\title{
Improvements of VIIRS and MODIS Solar Diffuser and Lunar Calibration
}

\author{
Xiaoxiong Xiong ${ }^{1}$, James Butler ${ }^{1}$, Ning Lei $^{2}$, Junqiang Sun ${ }^{2}$, Jon Fulbright ${ }^{2}$, \\ Zhipeng Wang ${ }^{2}$, Jeff McIntire ${ }^{2}$, and Amit Angal ${ }^{3}$ \\ ${ }^{1}$ Sciences and Exploration Directorate, NASA/GSFC, Greenbelt, MD 20771 \\ ${ }^{2}$ Sigma Space Corp., 4600 Forbes Boulevard, Lanham, MD 20706 \\ ${ }^{3}$ Science Systems and Applications Inc., 10210 Greenbelt Road, Lanham, MD 20706
}

\begin{abstract}
Both VIIRS and MODIS instruments use solar diffuser (SD) and lunar observations to calibrate their reflective solar bands (RSB). A solar diffuser stability monitor (SDSM) is used to track the SD on-orbit degradation. On-orbit observations have shown similar wavelength-dependent SD degradation (larger at shorter VIS wavelengths) and SDSM detector response degradation (larger at longer NIR wavelengths) for both VIIRS and MODIS instruments. In general, the MODIS scan mirror has experienced more degradation in the VIS spectral region whereas the VIIRS rotating telescope assembly (RTA) mirrors have seen more degradation in the NIR and SWIR spectral region. Because of this wavelength dependent mirror degradation, the sensor's relative spectral response (RSR) needs to be modulated. Due to differences between the solar and lunar spectral irradiance, the modulated RSR could have different effects on the SD and lunar calibration. In this paper, we identify various factors that should be considered for the improvements of VIIRS and MODIS solar and lunar calibration and examine their potential impact. Specifically, we will characterize and assess the calibration impact due to SD and SDSM attenuation screen transmission (uncertainty), SD BRF uncertainty and onorbit degradation, SDSM detector response degradation, and modulated RSR resulting from the sensor's optics degradation. Also illustrated and discussed in this paper are the calibration strategies implemented in the VIIRS and MODIS SD and lunar calibrations and efforts that could be made for future improvements.
\end{abstract}

Keywords: MODIS, VIIRS, radiometer, calibration, solar diffuser, solar diffuser stability monitor

\section{INTRODUCTION}

The Moderate Resolution Imaging Spectroradiometer (MODIS) is currently operated on-board the Terra spacecraft launched in December 1999 and Aqua spacecraft launched in May 2002. MODIS is a cross-track scanning radiometer that uses a double-sided scan mirror. It has 36 spectral bands, 20 reflective solar bands (RSB) and 16 thermal emissive bands (TEB), covering wavelengths from 0.41 to $14.5 \mu \mathrm{m}$. The RSB, with wavelengths from 0.41 to $2.2 \mu \mathrm{m}$, are calibrated by a solar diffuser (SD) and a solar diffuser stability monitor (SDSM). In addition, regularly scheduled lunar observations are made to track RSB calibration stability ${ }^{1-3}$. The Visible/Infrared Imager Radiometer Suite (VIIRS) sensor currently operated on-board the Suomi National Polar-orbiting Partnership (S-NPP) spacecraft was launched in October 2011. It was designed and built with a strong MODIS heritage. Instead of a scan mirror, the VIIRS sensor uses a rotating telescope assembly (RTA) to collect data from the earth view (EV) and the on-board calibrators (OBC). VIIRS

Earth Observing Systems XVIII, edited by James J. Butler, Xiaoxiong Xiong, Xingfa Gu, Proc. of SPIE Vol. 8866, 88661M · C 2013 SPIE · CCC code: 0277-786X/13/\$18 · doi: 10.1117/12.2024792 
makes observations in 22 spectral bands, $14 \mathrm{RSB}, 7 \mathrm{TEB}$, and 1 day night band (DNB), covering wavelengths from 0.41 to $12.4 \mu \mathrm{m}$. Its RSB, covering similar wavelength range as MODIS, are also calibrated by the SD and lunar observations $^{4-6}$. Table 1 is a comparison of VIIRS and MODIS RSB spectral range and their nadir spatial resolution. VIIRS M1-M5 and M7 are the dual gain bands.

Table 1 VIIRS and MODIS bands spectral range and nadir spatial resolution (HSR: horizontal sampling resolution). M1-M5 and M7 are dual gain bands.

\begin{tabular}{|c|c|c|c|c|c|}
\hline VIRS Band & Spectral Range (um) & Nadir HSR (m) & MODIS Band(s) & Range & HSR \\
\hline DNB & $0.500-0.900$ & & & & \\
\hline ○ M1 & $0.402-0.422$ & 750 & 8 & $0.405-0.420$ & 1000 \\
\hline ONR & $0.436-0.454$ & 750 & 9 & $0.438-0.448$ & 1000 \\
\hline$O M B$ & $0.478-0.498$ & 750 & 10 & $\begin{array}{l}0.459-0.479 \\
0.483-0.493\end{array}$ & $\begin{array}{c}500 \\
1000\end{array}$ \\
\hline$O \mathrm{Mm}$ & $0.545-0.565$ & 750 & 4 or 12 & $\begin{array}{l}0.545-0.565 \\
0.546-0.556\end{array}$ & $\begin{array}{c}500 \\
1000\end{array}$ \\
\hline 11 & $0.600-0.680$ & 375 & 1 & $0.620-0.670$ & 250 \\
\hline$\bigcirc$ ME & $0.662-0.682$ & 750 & 13 or 14 & $\begin{array}{l}0.662-0.672 \\
0.673-0.683\end{array}$ & $\begin{array}{l}1000 \\
1000\end{array}$ \\
\hline M6 & $0.739-0.754$ & 750 & 15 & $0.743-0.753$ & 1000 \\
\hline D & $0.846-0.885$ & 375 & 2 & $0.841-0.876$ & 250 \\
\hline O MI & $0.846-0.885$ & 750 & 16 or 2 & $\begin{array}{l}0.862-0.877 \\
0.841-0.876 \\
\end{array}$ & $\begin{array}{c}1000 \\
250 \\
\end{array}$ \\
\hline$M+8$ & $1230-1250$ & 750 & 5 & SAME & 500 \\
\hline MA & $1.371-1.386$ & 750 & 26 & $1.360-1.390$ & 1000 \\
\hline $\mathbf{B}$ & $1.580-1.640$ & 375 & 6 & $1.628-1.652$ & 500 \\
\hline M10 & $1.580-1.640$ & 750 & 6 & $1.628-1.652$ & 500 \\
\hline M11 & $2225-2275$ & 750 & 7 & $2.105-2.155$ & 500 \\
\hline
\end{tabular}

On-orbit SDSM observations have found that the SD degradations for both MODIS and VIIRS have similar wavelengthdependent features with larger degradations occurring at shorter visible (VIS) wavelengths ${ }^{7-11}$. Meanwhile, the SDSM detector responses have also experienced wavelength-dependent degradation, which is larger at longer near-infrared (NIR) wavelengths ${ }^{12}$. For MODIS, the double-sided scan mirror has shown more optical degradation in the VIS spectral region. This degradation is wavelength, mirror side, and mirror scan-angle dependent ${ }^{7-8}$. Due to coating contamination, VIIRS rotating telescope assembly (RTA) mirrors have experienced more degradation in the NIR and SWIR spectral region $^{13-15}$. Because of this wavelength-dependent optics degradation, the sensor's relative spectral response (RSR) or spectral response function (SRF), combined with in-band (IB) and out-of-band (OOB) responses, needs to be adjusted or modulated. It is expected that the modulated RSR will impact the solar and lunar calibration differently due to the differences between the solar and lunar spectra.

In this paper, we briefly describe VIIRS and MODIS solar and lunar calibration approaches, identify various factors that have been and/or should be considered to improve RSB on-orbit calibration accuracy. Specifically, the effects due to SD and SDSM attenuation screen transmission (uncertainty), SD BRF and its on-orbit degradation, sensor optics degradation modulated RSR, and SDSM detector (response) degradation are analyzed and discussed. Also presented in this paper are lessons from MODIS and VIIRS calibration and characterization. It should be pointed out that some of the specific topics have also been addressed in separate papers in the same proceedings ${ }^{11,16-17}$ and most of issues identified will be continuously studied for future improvements. 


\section{MODIS AND VIIRS SOLAR DIFFUSER AND LUNAR CALIBRATION}

Details of MODIS SD and lunar calibration methodologies have been described in a number of references ${ }^{6-8,} 10,15,18-19$. As shown in Figure 1, MODIS RSB calibration coefficients $\left(\mathrm{m}_{1}\right)$, which are inversely proportional to detector gains, are derived from the $\mathrm{SD}$ observations. In addition to the $\mathrm{SD} \mathrm{BRF}\left(\mathrm{BRF}_{\mathrm{SD}}\right)$ and its on-orbit change or degradation $\left(\Delta_{\mathrm{SD}}\right)$, the calibration coefficient also corrects for the Earth-Sun distance $\left(\mathrm{d}_{\text {Earth-Sun }}\right)$ and solar zenith angle $\left(\theta_{\mathrm{SD}}\right)$. For the bands that are calibrated with the SD screen in place, a screen transmission or vignetting function $\left(\Gamma_{\mathrm{SD}}\right)$ is considered. The detector responses to the $\mathrm{SD}\left(\mathrm{dn}_{\mathrm{SD}}\right)$ are corrected for instrument background, temperature, and scan angle difference. By design, the on-orbit change in SD BRF $\left(\Delta_{\mathrm{SD}}\right)$ is determined from SDSM observations. The SDSM is a ratioing radiometer that uses the same optics, detectors, and electronics to collect data from its Sun view through an attenuation screen, the SD view, and the dark view (for background measurements). In the expression for $\Delta_{\mathrm{SD}}$, the $\mathrm{dc}_{\mathrm{SD}}$ and $\mathrm{dc}_{\mathrm{Sun}}$ (digital counts for SDSM's SD and Sun views) are corrected for the background and screen transmission as needed. The spectral range covered by the MODIS SDSM detectors (1-9) is from 0.41 to $0.94 \mu \mathrm{m}$. Similarly, MODIS lunar calibration coefficients are determined from detector responses to the Moon $\left(\mathrm{dn}_{\text {Moon }}\right)$. The $f$ factor in the lunar calibration equation shown in Figure 1 includes viewing geometry corrections for the lunar phase/libration angles and Sun-Moon and Moon-Sensor distances.

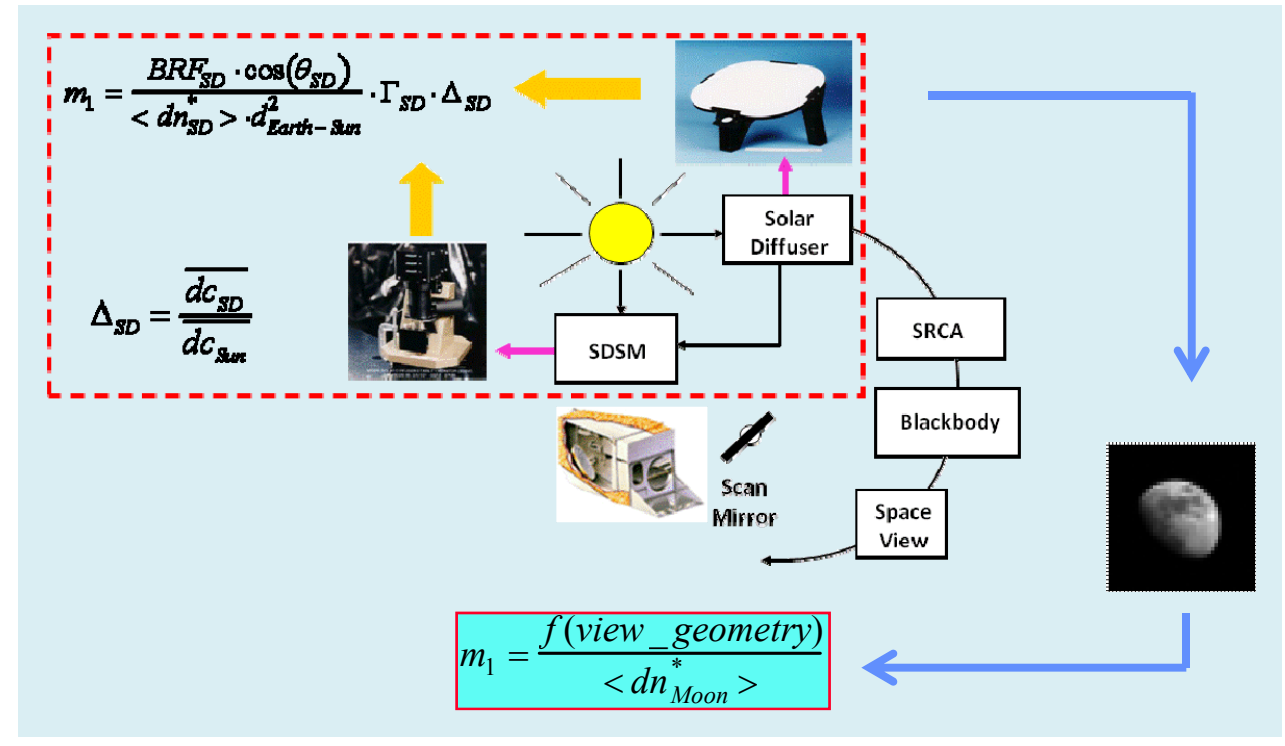

Figure 1 MODIS solar diffuser and lunar calibration schematic.

VIIRS SD and SDSM calibration system was built and improved based on lessons learned from MODIS. As expected, the VIIRS RSB solar and lunar calibration approach is adapted from MODIS experience. In VIIRS, the calibration coefficients and SD degradation are denoted as the F factors $(\mathrm{F})$ and $\mathrm{H}$ factors $(\mathrm{H})$, respectively. The $\mathrm{F}$ is a scaling factor derived from the ratio of the computed (or expected) solar radiance reflected from the SD panel to the sensor measured radiance using the pre-launch calibration coefficients $\left(\mathrm{c}_{\mathrm{i}}\right)$. Like MODIS, other correction factors, such as instrument temperature and scan angle difference for the half angle mirror (HAM), are also applied. In principle, VIIRS H factor is determined the same way as MODIS $\Delta_{\mathrm{SD}}$. The VIIRS SDSM has 8 detectors, covering a similar spectral range to the MODIS SDSM. Using VIIRS lunar observations, an equivalent F-factor can also be derived via integrated lunar irradiance measured by the sensor and that predicted by the lunar model ${ }^{20}$. Various details relating to the VIIRS SD calibration are shown in Figure 2.

MODIS SD calibration is performed on a regular basis. The SDSM is operated during each SD calibration event to track SD on-orbit degradation. When no SD calibration is scheduled, the SD door is placed into the closed position. MODIS SD calibration can be made with or without an attenuation screen, depending on the bands with high or low gains that 
saturate or do not saturate when the screen is at the open position. At the beginning of each mission, MODIS SD/SDSM calibration was performed on a weekly basis and then its frequency was gradually reduced. VIIRS has a fixed solar attenuation screen in front of the SD panel but it does not have an SD door. As a result, VIIRS SD calibration is performed every orbit. Currently, the VIIRS SDSM is operated on a daily basis.

MODIS lunar observations are made through its space view (SV) port at the "same" phase angles, $55^{\circ}$ to $56^{\circ}$ for Terra MODIS and $-55^{\circ}$ to $-56^{\circ}$ for Aqua MODIS. For this purpose, a spacecraft roll maneuver is often required. To collect more data samples during lunar observations, a sector rotation is made so that the lunar data (images) are stored in the EV data sector. VIIRS lunar observations are also made through its SV port, which is an extension of the EV port. Due to spacecraft operation and roll angle constraints, the VIIRS lunar observations are currently made with phase angles from $-51.5^{\circ}$ to $-50.5^{\circ}$. Like MODIS, during each scheduled lunar calibration event, a sector rotation is also made to store the lunar data in the EV data sector. Table 2 is a summary of Terra and Aqua MODIS and VIIRS lunar calibration events on a yearly basis.

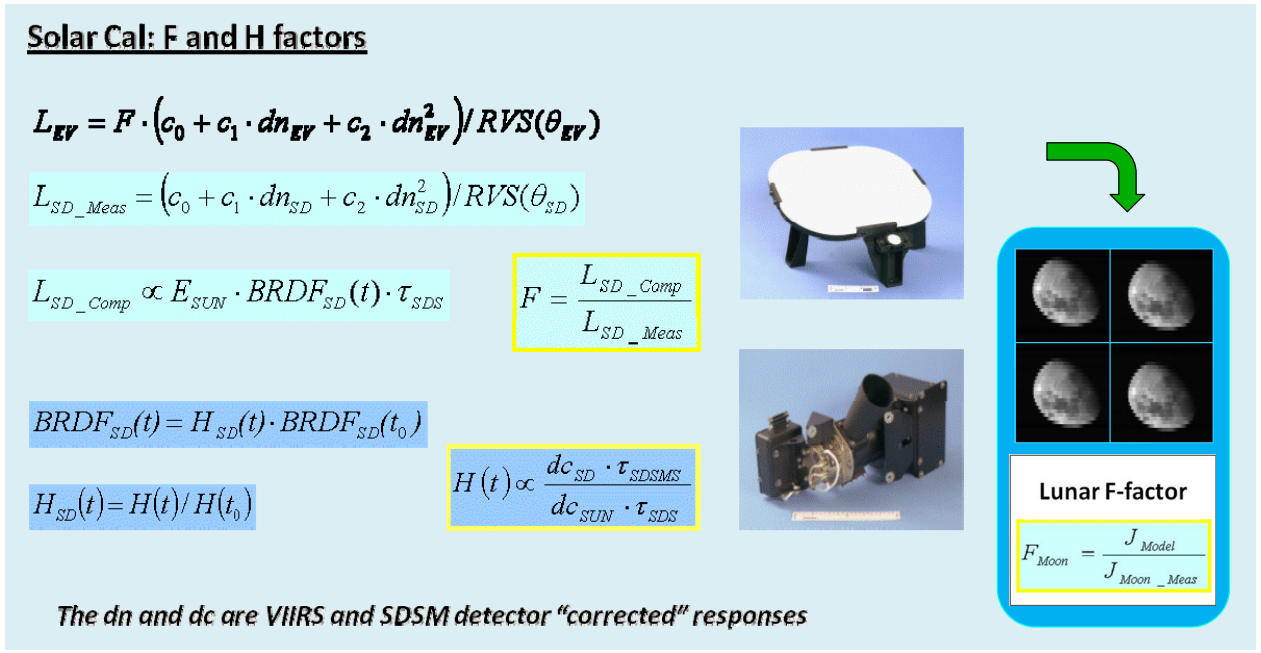

Figure 2 VIIRS solar diffuser and lunar calibration schematic.

Table 2 Scheduled lunar observations for Terra and Aqua MODIS and SNPP VIIRS (as of Aug. 2013)

\begin{tabular}{|c|c|c|c|c|c|c|c|c|c|c|c|c|c|c|}
\hline Year & 2000 & 2001 & 2002 & 2003 & 2004 & 2005 & 2006 & 2007 & 2008 & 2009 & 2010 & 2011 & 2012 & 2013 \\
\hline Terra MODIS & 9 & 10 & 10 & 9 & 10 & 8 & 9 & 9 & 10 & 8 & 9 & 8 & 10 & 7 \\
\hline Aqua MODIS & & & 5 & 9 & 10 & 9 & 9 & 7 & 9 & 11 & 10 & 8 & 10 & 6 \\
\hline SNPP VIIRS & & & & & & & & & & & & & 8 & 5 \\
\hline
\end{tabular}

\section{KEY FACTORS FOR CALIBRATION IMPROVEMENTS}

\subsection{SD and SDSM Screen}

The SDSM is used to track on-orbit changes in SD BRF. This is accomplished using the ratios of SDSM SD view responses to its Sun view responses. Both MODIS and VIIRS SDSM view the Sun through an attenuation screen. Its transmission function (or vignetting function) is used when computing the ratios of the SDSM SD view responses to its Sun view responses. MODIS did not perform pre-launch characterization for its SDSM screen transmission. It had to use measurements made during spacecraft yaw maneuvers to derive the transmission function. As an improvement, VIIRS performed pre-launch characterization of its SDSM screen transmission. On-orbit yaw maneuvers were performed to validate and/or improve the pre-launch determined vignetting function (VF). Figure 3 illustrates Terra MODIS and SNPP VIIRS SDSM Sun view responses from their individual detectors. Due to a design error in the MODIS SDSM sub- 
system, large variations existed in its Sun view responses. As a result, an alternative approach was developed by the MODIS Characterization Support Team (MCST) to normalize individual detector responses to those from detector 9 as the SD degradation at this wavelength $(0.94 \mu \mathrm{m})$ is expected to be extremely small and the patterns in all the detectors are nearly identical ${ }^{9,12}$. In the VIIRS SDSM design, this defect was eliminated. As expected, the variations in the VIIRS SDSM Sun view responses are much smaller. In addition to SDSM screen transmission, MODIS used its SD observations during yaw maneuvers to characterize its SD screen transmission and validate the SD BRF determined from pre-launch measurements ${ }^{21}$.

For VIIRS, the yaw data were used to improve its SDSM screen transmission and the product of SD screen VF and SD BRF. Unlike MODIS, the SD screen in VIIRS is fixed, so the SD observations cannot be used to characterize the SD screen VF and SD BRF separately. Figure 4 (left) shows the ratios of VIIRS SDSM screen pre-launch VF (detector 1) to on-orbit VF derived from yaws. The relative differences between the two VFs vary, depending on the solar illumination angles. The actual calibration range is typically smaller than the VF range shown in Figure 4 (left). The calibration impact due to differences between the two VFs, or the VF relative uncertainty, can be assessed by comparing the SD degradation (H-factor) derived from two different VF look-up tables (LUTs) shown in Figure 4 (right). The same approach can also be used to assess the impact due to uncertainties in SD VF.
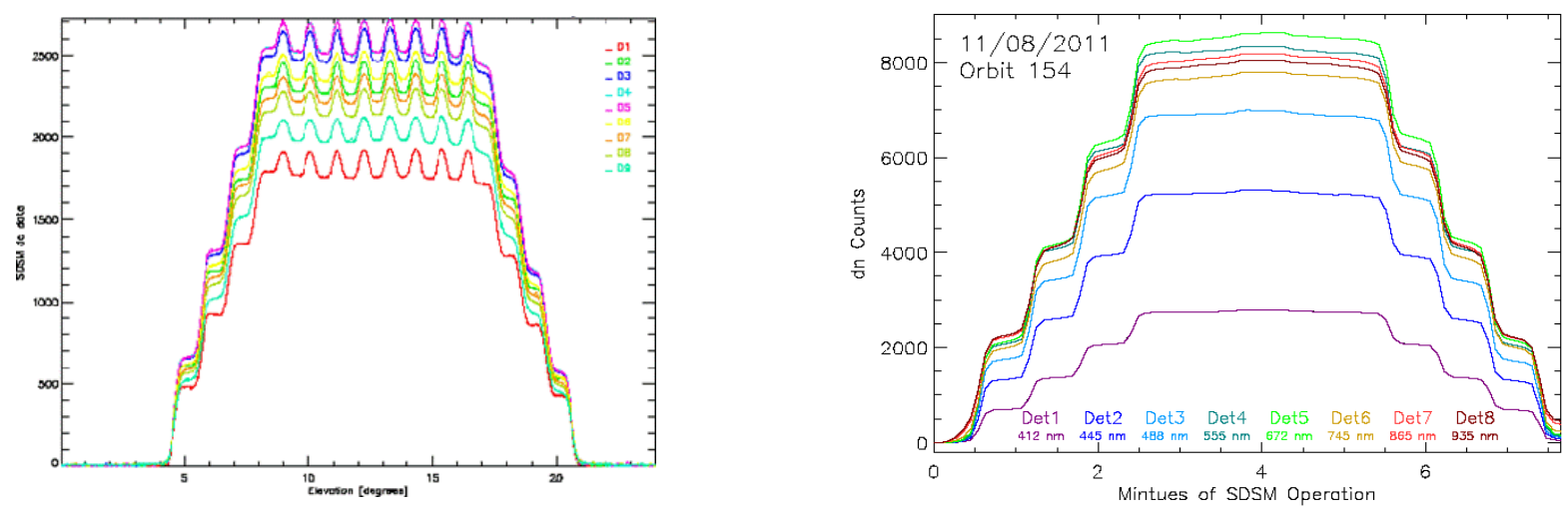

Figure 3 Left: Terra MODIS SDSM Sun view responses (9 detectors; data from year 2000 yaw maneuvers). Right: S-NPP VIIRS SDSM Sun view responses (8 detectors; data from SDSM calibration on Nov 08, 2011).
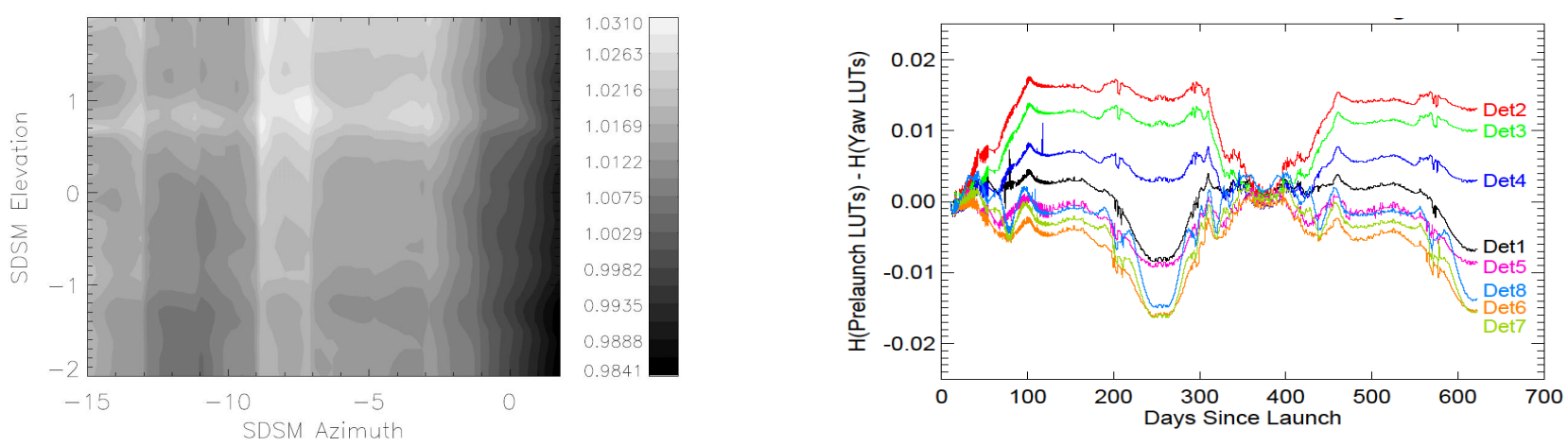

Figure 4 Left: Ratios of VIIRS SDSM screen VF derived from pre-launch characterization to that derived from on-orbit yaw data (detector 1). Right: Differences between VIIRS SDSM H-factors derived from pre-launch VF LUTs and that derived from on-orbit VF LUTs. 
It should be noted that the effect caused by the uncertainties of the SDSM screen VF, SD screen VF, and SD BRF usually appears in the form of periodic or seasonal patterns (features) in the calibration time series, as illustrated in Figure 4 (right). Apart from a constant offset, the calibration impact due to seasonal patterns is more severe at the mission beginning (short-term). A smooth function fitting to the long-term trending (or time series) can significantly reduce this type of impact. Currently, VIIRS uses detector dependent SDSM screen VF and spectral band dependent product of SD BRF and SD screen VF. In MODIS, a common SD VF is used for the bands that are calibrated with the screen in place. Future effort will be made to improve MODIS SD/SDSM calibration by re-examining and improving the SD and SDSM screen VF characterization.

\subsection{SDSM Detector Degradation}

The SDSM uses the Sun as the reference source to track SD BRF degradation. MODIS SDSM has 9 filtered detectors and VIIRS has 8, covering the same wavelength range from 0.41 to $0.94 \mu \mathrm{m}$ (see Table 3). On-orbit observations show that the SDSM detector response degrades over time. Shown in Figure 5 are Aqua MODIS and S-NPP VIIRS SDSM Sun view responses over time, normalized to their initial on-orbit measurements and corrected for different solar illumination conditions. As previously discussed, the VIIRS SDSM Sun view responses are much smoother than MODIS. From Figure 5, it is obvious that the SDSM Sun view response trending has a strong wavelength-dependent feature for both MODIS and VIIRS. This wavelength dependent response degradation, larger at longer (NIR) wavelengths, is likely due to on-orbit displacement damage to the detectors by the high-energy protons in the space environment ${ }^{22}$. Because of its wavelength-dependence, this degradation could affect the RSR of SDSM detectors and potentially impact the accuracy to track SD on-orbit degradation. Currently, the SDSM calibration only uses the center wavelength for each SDSM detector and thus does not include the effect due to SDSM detector degradation. Since the same SDSM detector system is used to view both the Sun and SD, the detector degradation effect on SD degradation computation should be relatively small. For the detectors with very small OOB response, this effect can be ignored.

Table 3 MODIS and VIIRS solar diffuser stability monitor (SDSM) detectors and wavelengths (in $\mu \mathrm{m}$ )

\begin{tabular}{|c|c|c|c|c|c|c|c|c|c|}
\hline SDSM Detector & D1 & D2 & D3 & D4 & D5 & D6 & D7 & D8 & D9 \\
\hline MODIS & 0.412 & 0.466 & 0.53 & 0.554 & 0.646 & 0.747 & 0.857 & 0.904 & 0.936 \\
\hline VIIRS & 0.412 & 0.445 & 0.488 & 0.555 & 0.672 & 0.746 & 0.865 & 0.935 & \\
\hline
\end{tabular}
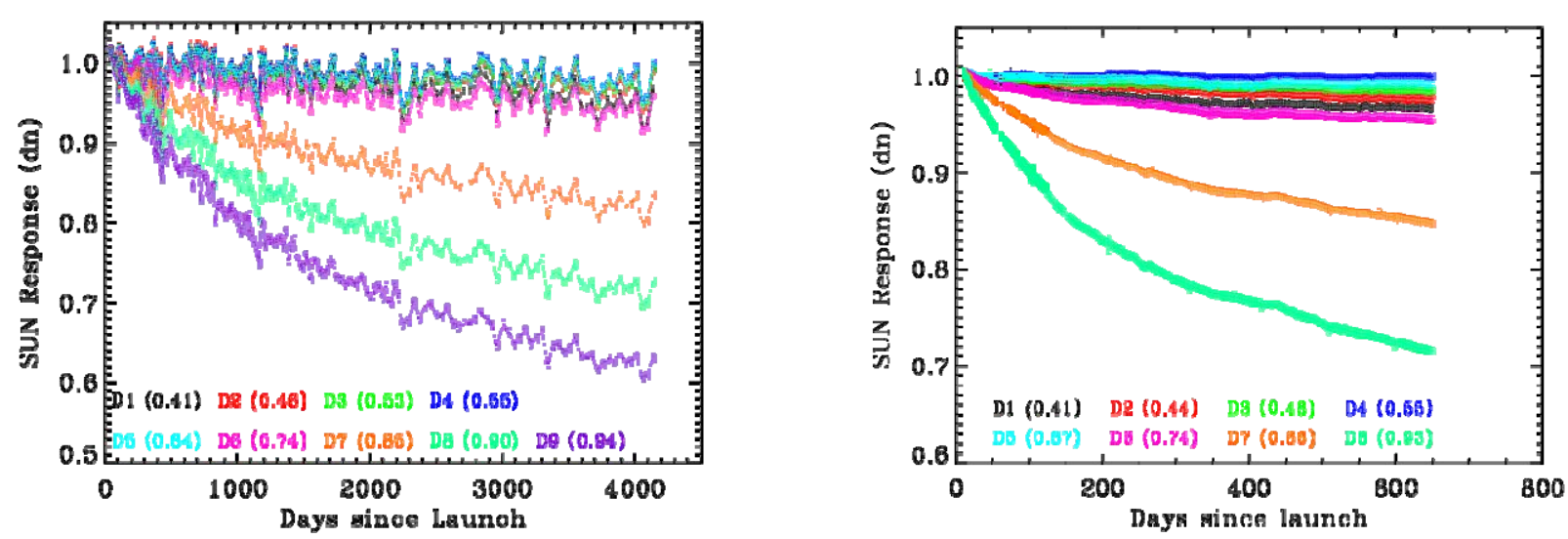

Figure 5 Aqua MODIS (left) and S-NPP VIIRS (right) SDSM Sun view responses (normalized and corrected for solar illumination conditions).

It was discovered shortly after the S-NPP launch that VIIRS SDSM detector filters could have small but non-negligible OOB response, depending on how the filters are orientated (or placed in front of the detectors). The same problem might also exist in MODIS SDSM. Since no SDSM assembly level RSR characterization was made before launch, it is difficult 
to have an accurate calibration impact assessment. Because of its potential calibration impact, the SD on-orbit degradation is carefully monitored and analyzed for both MODIS and VIIRS. As mission continues, the lunar calibration trending could be used to study this effect for VIIRS as SD and lunar observations are made at the same scan angle.

\subsection{SD BRF and On-orbit Degradation}

MODIS and VIIRS SD BRF characterization was made pre-launch using reference samples traceable to NIST reflectance standards. On-orbit changes in SD BRF are determined by the SDSM. Figure 6 shows Aqua MODIS and SNPP VIIRS SD degradation over time and Figure 7 presents their current degradation as a function of wavelength. In general, MODIS and VIIRS SD degradation has the same wavelength dependent degradation with larger degradation at shorter wavelengths. Although Aqua MODIS has operated more than 11 years and S-NPP VIIRS less than 2 years, VIIRS SD has already experienced more degradation. This is mainly due to the fact that VIIRS SD is illuminated by the Sun every orbit. It is expected that the degradation rate will gradually decrease as the mission continues. The strong wavelength dependent behavior needs to be carefully considered when computing the SD degradation $\left(\Delta_{\mathrm{SD}}\right.$ in MODIS or $\mathrm{H}$-factor in VIIRS) and sensor calibration coefficients ( $\mathrm{m}_{1}$ in MODIS or F-factor in VIIRS).
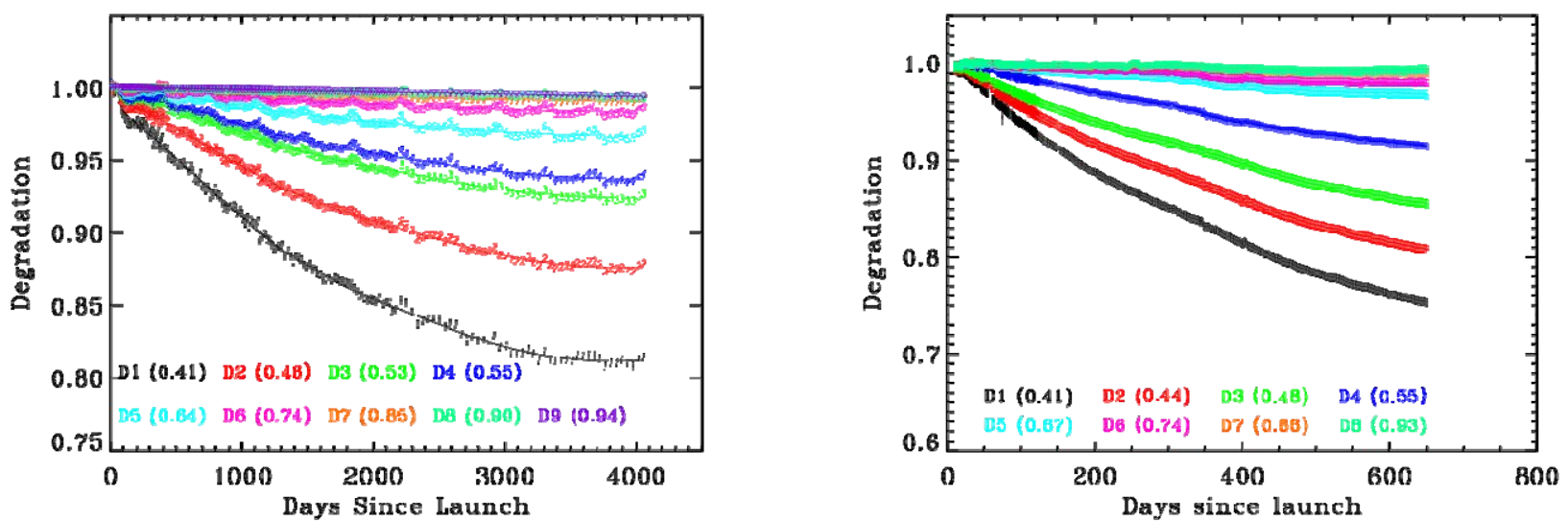

Figure 6 Aqua MODIS (left) and SNPP VIIRS (right) SD on-orbit degradation time series.

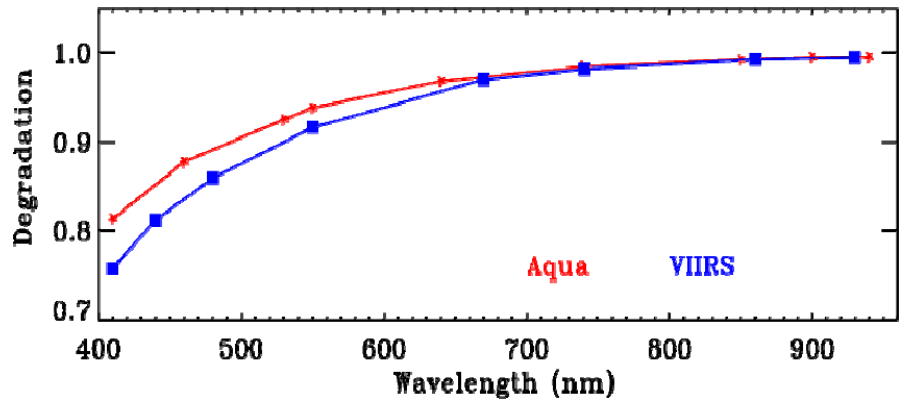

Figure 7 Aqua MODIS (left) and SNPP VIIRS (right) SD on-orbit degradation as a function of wavelength.

If the SDSM detector system OOB response is very small, then the impact can generally be ignored. Otherwise, the SD degradation as a function of wavelength should be integrated together with the solar spectral irradiance and the RSR. Alternatively, one can think of this effect as the modulation of solar spectrum when the Sun light is reflected from the SD panel. Because of this, there is a mismatch of the two solar spectra seen by SDSM detector system, one directly from the Sun (through an attenuation screen) and the other reflected by the SD. For the same reason that the SDSM assembly level RSR was not characterized, an accurate impact assessment remains challenging. 
On the other hand, MODIS and VIIRS detector RSR functions, in-band and out-of-band combined, were fully characterized pre-launch. Depending on the (spectral) location and (response) magnitude of the OOB, the SD degradation could have some impact when deriving the calibration coefficients. Currently, the H-factor as a function of wavelength is used to compute the F-factor for VIIRS. As demonstrated in Figure 8, the SD degradation impact on VIIRS VIS/NIR calibration could be as high as $0.5 \%$ for band M1. For other bands, the potential impact is generally less than $0.2 \%$. Comparing MODIS and VIIRS detector RSR functions indicates that this effect in MODIS calibration should be smaller. Examples of RSRs for MODIS band 8 and VIIRS M1 as well as MODIS band 1 and VIIRS I1 are presented in Figure 9. Currently, MODIS calibration algorithm has not included the OOB RSR when computing its calibration coefficients. Future effort could be made to address this issue and to improve its RSB (SD) calibration.

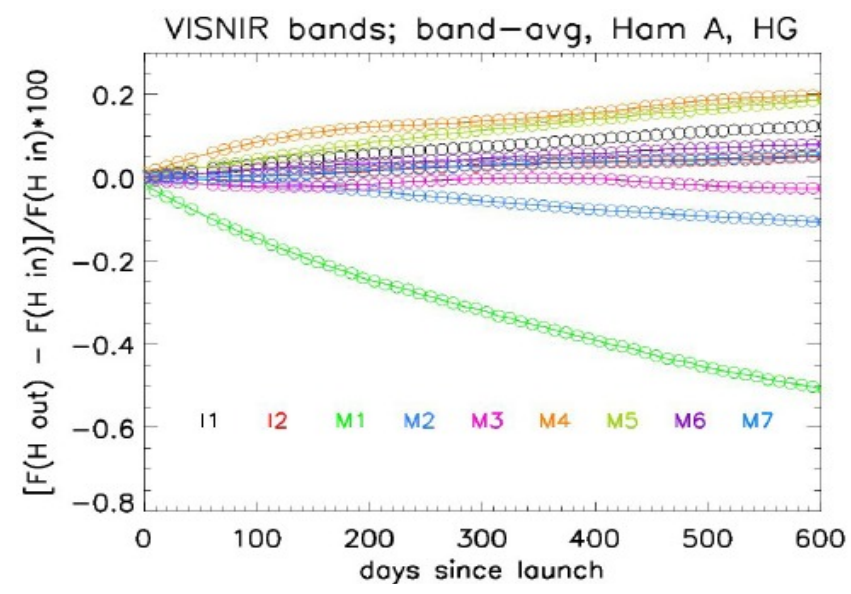

Figure 8 SD degradation impact on calibration coefficients.
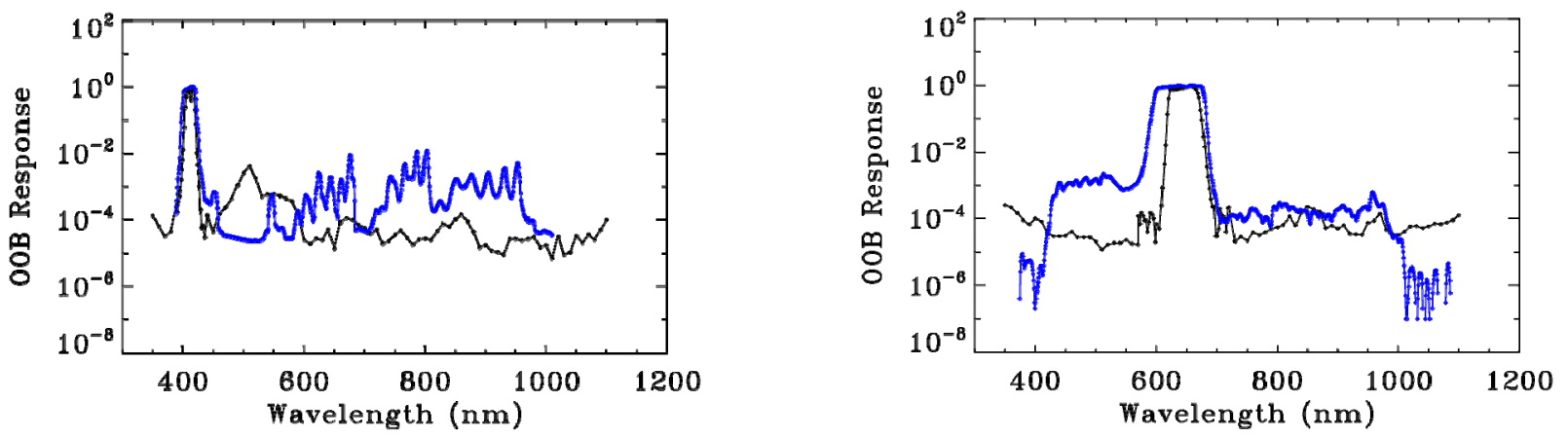

Figure 9 Aqua MODIS (black curve) and SNPP VIIRS (blue curve) RSR for band 8 and M1 (left) and band 1 and I1 (right).

\subsection{Optics Degradation}

Shortly after launch, it was found that VIIRS optical response had significantly degraded in the NIR and SWIR spectral regions. It was later identified that the wavelength dependent optics degradation was caused by the RTA mirror contamination $^{13-15}$. Illustrated in Figure 10 (left) is an example of VIIRS sensor response trending for bands M1-M5 and M7. Using a physics-based model to fit the detector responses, one can predict/estimate sensor future performance. Assuming most changes in detector responses are due to RTA optics degradation, Figure 11 shows the modeled/predicated RTA reflectance at year 1, year2, and year 7. Obviously, large changes occurred early in the mission. Based on the observations and model predication, it is expected that the degradation will gradually level off as mission continues. More details on the degradation model and fitting strategy can be found in other publications ${ }^{16,23}$. 
Because of the RTA mirror degradation, the sensor RSR (IB and OOB combined) determined from pre-launch characterization is modulated on-orbit. As previously described, the modulated RSR could impact the solar calibration as well as lunar calibration.

As a demonstration of modulated RSR impact on SD calibration, the differences between the F-factors derived from modulated RSR and that derived from the pre-launch RSR are presented in Figure 11 (left) for the VIIRS VIS/NIR spectral bands. Currently, the modulated RSRs have been applied in the VIIRS SDR (sensor data record) production and made available for the science and user community. Although the current calibration impact due to modulated RSR is relatively small for the VIS/NIR bands, a much larger effect is observed in the DNB bands. Displayed in Figure 11 (right) is the trending of VIIRS DNB low gain (LG) responses (gains or 1/F-factors) derived from on-orbit SD calibrations. One of trends is produced using fixed pre-launch RSR and the other using the modulated RSR. Since the DNB spectral range is from 0.5 to $0.9 \mu \mathrm{m}$, much broader than the M- and I-bands, and the largest RTA mirror degradation is at 0.9 to $1.0 \mu \mathrm{m}$, the impact due to modulated RSR on DNB has been more significant.
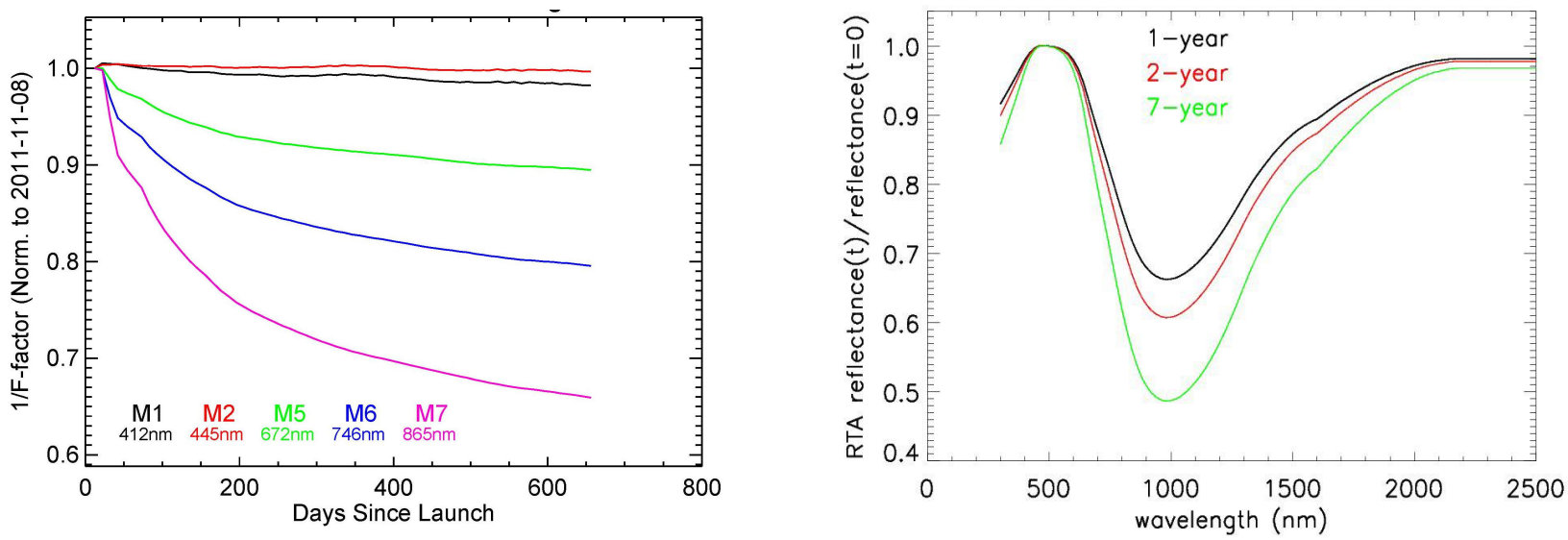

Figure 10 VIIRS normalized response or 1/F-factor trending (left) and modeled changes as a function of wavelengths.
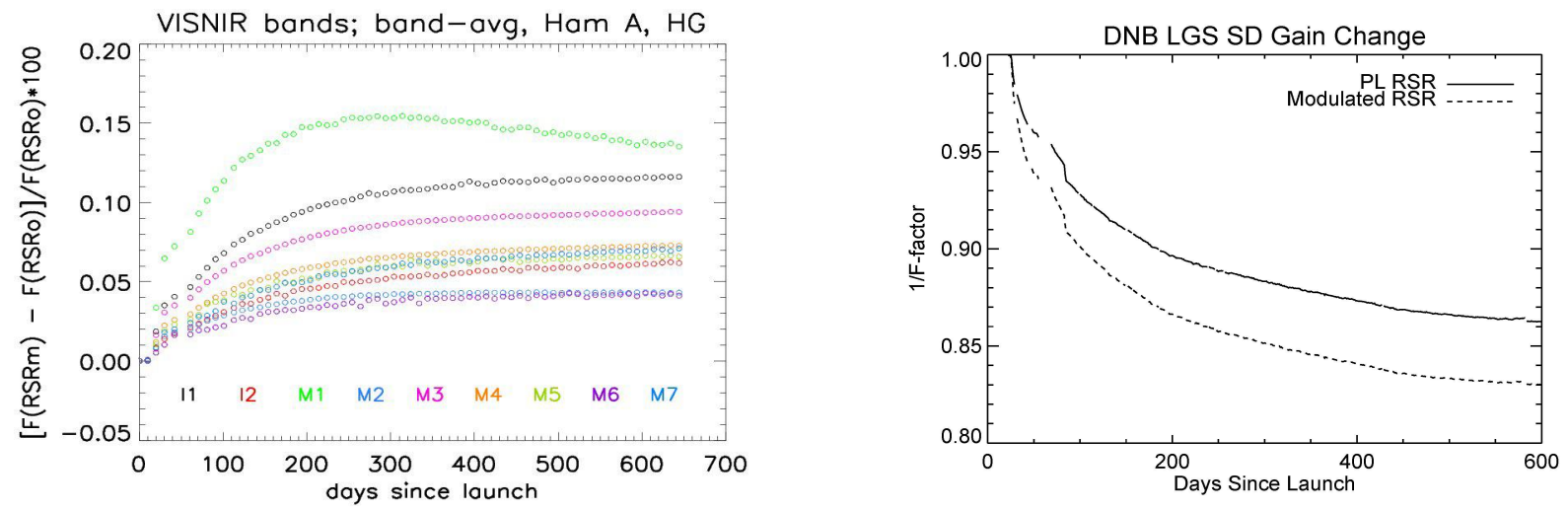

Figure 11 Modulated RSR impact on VIIRS VIR/NIR F-factors (left) and comparison of DNB low gain responses (1/Ffactor) derived from the pre-launch RSR and that from the modulated RSR.

For VIIRS, lunar observations can be used to derive an equivalent F-factor or to compute the lunar irradiance (integrated) using calibration coefficients derived from the SD calibration. In both cases, the effects due to modulated RSR need to be examined and addressed if necessary. In case 1 (to derive an equivalent F-factor), a model predicated lunar irradiance is used to correct lunar viewing geometry differences. The sensor-specific RSR is needed to calculate the predicated lunar irradiance. Figure 12 illustrates the impact due to modulated RSR on VIRIS bands M1-M3 
calibration. In case 2, calibration coefficients used to compute lunar irradiance are impacted by the modulated RSB. Like SD calibration, the modulated RSR impact is much larger on DNB lunar calibration.

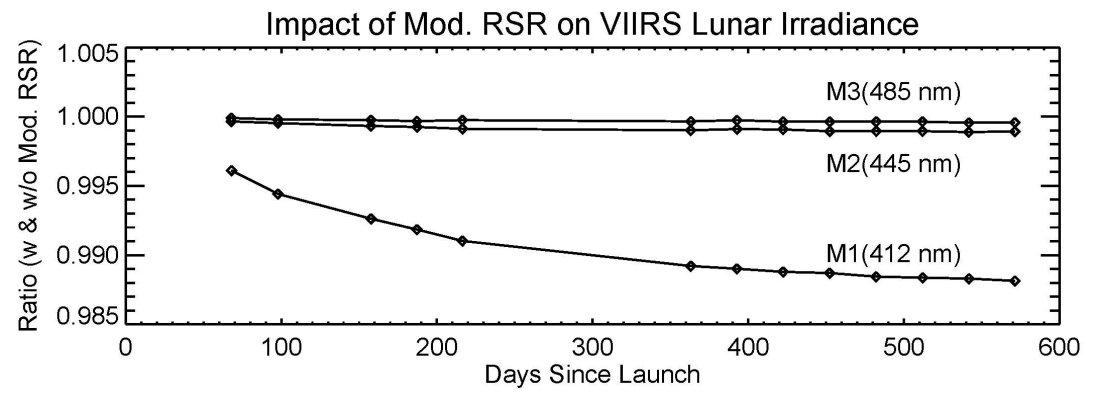

Figure 12 Radios of VIIRS lunar irradiance computed using modulated RSR to that computed with fixed (non-modulated) RSR for bands M1-M3. (lunar irradiance provided by the ROLO model ${ }^{20}$ )

For MODIS, the modulated RSR effects for RSB calibration should be relatively small (see Figure 9 and discussions in section 3.3). The current MODIS calibration algorithm has not included the OOB RSR, thus no modulated RSR effect is considered. For future improvements, more in-depth analyses will be carried out.

\section{SUMMARY}

This paper provides a brief description of MODIS and VIIRS SD and lunar calibration methodologies and activities. It identifies key factors that should be considered to assure their reflective solar bands (RSB) on-orbit calibration quality, which include SD and SDSM screen vignetting function, SD BRF and its on-orbit degradation, and modulated RSR due to optics degradation. Most of issues have been studies by members of MODIS Characterization Support Team (MCST) and VIRIS Characterization Support Team (VCST) with varying degrees of effort and success. As illustrated, the lessons learned from MODIS have greatly benefited the work in VIIRS and, similarly, progress made in VIIRS calibration and characterization has also helped the MODIS. Some of issues discussed here remaining challenging and will be continuously studied for better understanding of sensor performance and for future calibration improvements.

\section{REFERENCES}

1. W.L. Barnes and V.V. Salomonson, "MODIS: A global image spectroradiometer for the Earth Observing System," Critical Reviews of Optical Science and Technology, CR47, 285-307, 1993

2. W.L. Barnes, X. Xiong, and V.V. Salomonson, "Status of Terra MODIS and Aqua MODIS," J. of Advances in Space Research, 32/11, 2099-2106, 2003

3. X. Xiong, K. Chiang, J. Esposito, B. Guenther, and W.L. Barnes, "MODIS On-orbit Calibration and Characterization," Metrologia 40 89-92, 2003

4. Schueler, C. F., E. Clement, P. Ardanuy, C. Welsh, F. De Luccia, and H. Swenson, "NPOESS VIIRS sensor design overview," Proc. SPIE, vol. 4483, 11-23, 2002

5. Murphy, R.P., P. E. Ardanuy, F. Deluccia, J. E. Clement, and C. Schueler, "The visible infrared imaging radiometer suite, Earth Science Satellite Remote Sensing,” vol. 1, New York, USA: Springer-Verlag, pp. 199-223, 2006

6. Xiong, X., J. Butler, K. Chiang, B. Efremova, J. Fulbright, N. Lei, J. McIntire, H. Oudrari, J. Sun, Z. Wang, and A. $\mathrm{Wu}$, "VIIRS On-orbit Calibration Methodology and Performance," JGR (submitted)

7. Xiong X., J. Sun, W. Barnes, V. Salomonson, J. Esposito, H. Erives, and B. Guenther, "Multi-year On-orbit Calibration and Performance of Terra MODIS Reflective Solar Bands," IEEE Transactions on Geoscience and Remote Sensing, Vol. 45, No. 4, 879-889, 2007

8. X. Xiong, J. Sun, X. Xie, W. Barnes, and V. Salomonson, "On-Orbit Calibration and Performance of Aqua MODIS Reflective Solar Bands," IEEE Trans. Geosci. Remote Sens., 48(1), 535-546, 2010 
9. X. Xiong, X. Xie, A. Angal, J. Choi, J. Sun, and W.L. Barnes, "Characterization of MODIS Solar Diffuser On-orbit Degradation,” Proceedings of SPIE - Earth Observing Systems XII, Vol. 6677, 66770O, doi: 10.1117/12.731304, 2007

10. Fulbright, J., N. Lei, K. Chiang, X. Xiong, "Characterization and performance of the Suomi-NPP/VIIRS solar diffuser stability monitor," Proc. SPIE 8510, 851015, 2012

11. Fulbright, J., N. Lei, J. McIntire, B. Efremova, X. Chen, and X. Xiong, "Improving the Characterization and performance of the Suomi-NPP/VIIRS solar diffuser stability monitor," Proc. SPIE 8866, 2013

12. Xiong, X., J. Fulbright, A. Angal, J. Sun, and Z. Wang, "Comparison of MODIS and VIIRS Solar Diffuser Stability Monitor Performance", Proc. SPIE 8528, 852809, 2012

13. Barrie, J. D., P. D. Fuqua, M. J. Meshishnek, M. Ciofalo, C. Chu, J. Chaney, R. Moision, and L. Graziani, "Root cause determination of on-orbit degradation of the VIIRS rotating telescope assembly," Proc. SPIE, vol. 8510, 851009,2012

14. DeLuccia, F. J., D. Moyer, E. H. Johnson, K. W. Rausch, N. Lei, K. Chiang, X. Xiong, E. M. Haas, J. Fulbright, and G. Iona, "Discovery and characterization of on-orbit degradation of the VIIRS rotation telescope assembly," Proc. SPIE, vol. 8510, 85101A, 2012

15. Lei, N., Z. Wang, J. Fulbright., S. Lee, J. McIntire, K. Chiang, and X. Xiong, "Initial on-orbit radiometric calibration of the Suomi NPP VIIRS Reflective Solar Bands," Proc. SPIE, vol. 8510, 851018, 2012

16. Lei, N., Guenther, B., Wang, Z., and Xioing X., "Modeling SNPP VIIRS reflective solar bands optical throughput degradation and its impacts on the relative spectral response", SPIE Proc. Vol 8866, 2013

17. Lei, N., Wang, Z., Fulbright, J., and Xioing X., "Effect of the SDSM detector relative spectral response in determining the degradation of the SNPP VIIRS solar diffuser reflectance”, SPIE Proc. Vol 8866, 2013

18. Sun J, X. Xiong, W. Barnes, and B. Guenther, "MODIS Reflective Solar Bands On-orbit Lunar Calibration," accepted for IEEE Transactions on Geoscience and Remote Sensing, Vol. 45, No. 7, 2383-2393, 2007

19. Sun, J., X. Xiong, and J. Butler, "NPP VIIRS on-orbit calibration and characterization using the moon," Proc. SPIE, vol 8510, 85101I, 2012

20. Stone, T. and H. Kieffer, "An absolute irradiance of the Moon for on-orbit calibration", Proceedings of SPIE - Earth Observing Systems VII, 4814, 211-221, 2002

21. McIntire, J., B. Efremova, D. Moyer, S. Lee, and X. Xiong, "Analysis of Suomi-NPP VIIRS Vignetting Functions Based on Yaw Maneuver Data," Proc. SPIE, vol. 8510, 851001K, 2012

22. Xiong, X., A. Angal, T. Choi, J. Sun, and E. Johnson, "On-orbit performance of MODIS solar diffuser stability monitor," Proceedings of SPIE vol. 8510, issue $85100 \mathrm{H}, 2012$

23. Lei N., Z. Wang, B. Guenther, X. Xiong, and J. Gleason, "Modeling the detector radiometric response gains of the Suomi NPP VIIRS reflective solar bands," Proc. SPIE, vol. 8533, 853319, 2012 